How to reference this article

Peša Matracki, I. (2019). Aspetti pragmatici delle strategie evidenziali in italiano e in croato. Italica Wratislaviensia, 10(1), 179-205.

DOI: http://dx.doi.org/10.15804/IW.2019.10.1.8

Ivica Peša Matracki

Sveučilište u Zagrebu

ipesa@ffzg.hr, ORCID: 0000-0002-1292-3959

\title{
ASPETTI PRAGMATICI DELLE STRATEGIE EVIDENZIALI IN ITALIANO E IN CROATO
}

\section{PRAGMATIC ASPECTS OF EVIDENTIALS IN ITALIAN AND CROATIAN}

\begin{abstract}
Evidentials articulate the source of information on which a statement is based, which means that they also involve the speaker's position towards the sentence content. In this paper, after defining the subject of the research and describing various typologies of evidential strategies, we analyse the pragmatic values of linguistic expressions that articulate the various degrees of a speaker's involvement with regard to stated claims, which for their part represent speech acts used to endorse the veracity of a statement. Evidential strategies are frequently used to diminish responsibility for the veracity of claims, that is, they may serve as a means of distancing oneself from the sentence content, a discourse mechanism that the speaker may employ in order to avoid potential negative impacts that overly assertive claims might contain. In defining evidential strategies, we consult linguistic research as well as research on justification logic. The goal of this paper is to show that these terms can be adequately defined only by also taking into account the analysis of pragmatic impacts in a specific communicative and social situation.
\end{abstract}

Keywords: evidentials, pragmatic values, speech act, Italian, Croatian 


\section{PREMESSE TEORICHE}

T 'evidenzialità serve a esprimere la fonte o l'origine dell'informazio-

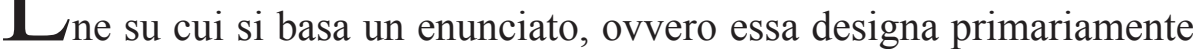
ed esplicitamente la fonte dell'informazione. La definizione implica che il concetto di evidenzialità sia a priori neutrale rispetto al giudizio del parlante sulla verità dell'enunciato. Il giudizio è espresso da un'altra categoria linguistica: la modalità epistemica. Da ciò consegue che gli evidenziali esprimono il tipo di fonte dell'informazione contenuta in un enunciato mentre la modalità epistemica valuta il grado di certezza o di probabilità delle asserzioni del parlante. ${ }^{1}$

Il dibattito sulle caratteristiche peculiari di questi due concetti e le corrispondenze tra loro negli ultimi decenni è piuttosto vivo e stimolante (cf. p. es. De Haan, 1999, 2001; Aikhenvald, 2004; Squartini, 2005; Mcready \& Norry, 2007; Diewald \& Smirnova, 2010)2. E anche se, secondo le nostre analisi, non è possibile stabilire corrispondenze biunivoche precise ed esclusive tra l'espressione della fonte e la valutazione della stessa, possiamo dire che tali concetti sono collegati perché l'esplicitazione della fonte dell'informazione in base alla quale si afferma qualcosa sovente rinforza l'attendibilità / veridicità / credibilità / fondatezza dell'enunciato, ossia, testimonia e/o (di)mostra che l'affermazione

1 Tale definizione è sostenuta, tra gli altri, da De Haan (1999, 2001) e Aikhenvald (2004). La parte teorica della presente indagine si appoggia principalmente sul lavoro di Peša Matracki \& Batinić Angster (2016) in cui è descritto in modo generale il quadro teorico che costituisce le premesse per l'analisi degli evidenziali in prospettiva semantica.

2 Studiando le affinità tra questi due concetti, non pochi linguisti affermano che gli evidenziali costituiscono una sottocategoria della modalità epistemica, ad esempio, Palmer (1986), McCready \& Norry (2007). De Haan (1999) invece sostiene che questi concetti differiscono sostanzialmente tra loro per l'origine, lo sviluppo e il comportamento sintattico; gli evidenziali semplicemente affermano che ci sono delle prove e testimonianze per le informazioni del parlante. Dunque, tutti i legami con la modalità epistemica sono secondari per natura: i due concetti codificano le entità diverse (source of information vs attitude towards that information, ivi, p. 8). 
pronunciata dal parlante è conforme al vero. ${ }^{3}$ In breve, la fonte spesso conferma e aumenta il grado di probabilità del contenuto proposizionale: Ho visto (fonte diretta visiva) che Vincenzo era distratto, distratto e pensieroso; Ti ho sentito (fonte diretta uditiva) mentre le dicevi che era difficile vivere con me; Leggendo questa frase ho sentito (fonte diretta sensoriale) un brivido attraversarmi il cuore.

Entrambi i concetti quindi riguardano l'affidabilità e la non causalità della verità contenuta nell'enunciato, cioè l'atteggiamento del parlante nei confronti della credenza (o dell'informazione) espressa. Le differenze invece tra i due concetti concernono soprattutto il modo in cui esprimono e valutano tale affidabilità. All'interno dell'evidenzialità il parlante specifica la fonte, il tipo e la valutazione dei fattori di verità del contenuto proposizionale mentre la modalità epistemica riguarda il contenuto proposizionale nei termini di probabilità/possibilità e di necessità (cf. Peša Matracki \& Batinić Angster, 2016, p. 29).

Nell'ambito degli studi linguistici più volte si è fatto ricorso a varie teorie filosofiche per descrivere i fenomeni di interesse generale. Nel nostro caso, i due concetti sono analizzabili tramite le seguenti nozioni della filosofia del linguaggio e della mente: $i$. natura o logica di giustificazione; $i i$. possesso non causale / aleatorio della verità (dell'enunciato); iii. condizione $X$ che rende affidabile il valore della verità espressa (la verità dell'enunciato va legata e assicurata con ragioni valide, ben fondate); $i v$. esperienza percettiva / processi inferenziali (credenze percettive dirette / credenze non direttamente percettive); $v$. possesso di dati evidenziali sufficienti (evidenza esterna / interna); vi. senso comune. ${ }^{4}$

${ }^{3}$ Per i risultati delle analisi condotte sui dati del corpus, si veda Peša \& Batinić Angster (2016).

${ }^{4}$ Queste nozioni costituiscono lo sfondo di ogni nostra credenza o conoscenza. Una delle ipotesi base della logica di giustificazione applicata (Applied Justification Logic) dice: il possesso della verità deve basarsi su ragioni giustificabili, $p(t: p)$; vuol dire che $p$ è giustificato con $t$. Dalla formula si vede che il soggetto detto agente (agens) possiede una prova/evidenza concreta (evidence), una giustificazione sufficiente per la verità sostenuta. Per ulteriori approfondimenti che giustificano quanto è detto sopra, v. Goldman (1986); Collin \& Guldmann (2005); Steup \& Sosa (2005); Livi (2013). 
Ci sono diversi modi in cui un parlante può indicare la testimonianza diretta o indiretta di quanto afferma: riflessione teoretica / attività raziocinante (speculation o supposition); procedimento logico / deduzione (inference); visto / sentito (sensory evidence); per sentito dire (hearsay); citazione (quotative). ${ }^{5}$ In base a questi modi, l'evidenzialità può essere analizzata come segue: $a$. fonti interne/dirette basate sulla percezione sensoriale, sull'inferenza o sul ragionamento (quindi di tipo congetturale-deduttivo o inferenziale) e $b$. fonti esterne/indirette (di tipo riportivo).

Nelle lingue senza sistema evidenziale di tipo grammaticale come l'italiano e il croato, l'espressione della fonte dell'informazione si svolge mediante mezzi lessicali e sintattici.

\section{VALORI PRAGMATICI DEGLI EVIDENZIALI}

Come abbiamo già osservato, dal punto di vista strettamente semantico, gli evidenziali segnalano che ci sono prove, testimonianze, attestazioni per il contenuto proposizionale. La prospettiva pragmatica fornisce invece diverse possibilità di interpretazione di tali testimonianze. ${ }^{6}$

In altre parole, con l'indicazione della fonte non si esauriscono le sfumature, le connotazioni e tantomeno il senso comunicativo ${ }^{7}$ di una strategia evidenziale, ma si specifica la forza pragmatica attraverso la quale il parlante produce gli effetti desiderati sui sentimenti, sui pensieri e sugli atteggiamenti dell'ascoltatore. Le strategie evidenziali riflettono inoltre le intenzioni con cui il parlante le ha utilizzate.

Poiché nelle due lingue esaminate l'espressione dell'origine dell'informazione è facoltativa, cioè si esplicita solo quando è ritenuto necessario, tramite questa espressione si vuole raggiungere uno scopo o ottenere un effetto. Da questo fatto consegue l'ipotesi di partenza (da verificare senza alcun preconcetto): gli evidenziali sono di per sé neu-

5 A nostro avviso (fondato sul lavoro di Palmer, 1986) il primo modo appartiene strettamente alla modalità epistemica mentre gli altri riguardano altresì l'evidenzialità.

${ }^{6}$ Queste possibilità sono in relazione con le convinzioni, le ragioni, i dubbi sulle nostre credenze / conoscenze nonché con il senso in cui il parlante valuta o presenta il contenuto vero-funzionale dell'enunciato.

7 Per la nozione di senso di Frege, si veda Dummett (1992). 
trali nei confronti del giudizio del parlante sulla verità proposizionale, e quando sono esplicitati/usati assumono diversi valori pragmatici, cioè implicano determinate interpretazioni all'interno di una concreta situazione comunicativa o scambio linguistico ${ }^{8}$.

L'analisi dell'uso degli evidenziali avrebbe dunque il compito di strutturare delle aree dei valori pragmatici (connessi all'uso dell'una o dell'altra strategia evidenziale) dalle quali conseguono valutazioni / intenzioni / adattamenti come parte di ciò che il parlante intende comunicare (come obiettivo comunicativo-situazionale) e come parte di ciò che il destinatario può percepire e comprendere nella concreta situazione comunicativa (al di là del valore semantico puro). ${ }^{9}$ I concetti che permettono il passaggio dal significato strettamente linguistico degli evidenziali al loro uso e al senso conversazionale sono relativi agli atti linguistici. ${ }^{10}$ Le difficoltà scaturiscono dal fatto che questi ultimi sono influenzabili dal contesto, ossia dall'insieme degli aspetti comunicativi sovente non definibili con precisione. La forza pragmatica degli evidenziali si esprime soprattutto nell'illocuzione e nella perlocuzione (cioè tramite un atto illocutorio e un atto perlocutorio). ${ }^{11}$

Per quanto riguarda gli aspetti teorici, in questo lavoro, seguiamo la divisione degli atti illocutori di Searle (1969, p. 66): richiedere (request), asserire (assert), domandare (question), ringraziare (thank), consigliare (advise), ammonire (warn), salutare (greet), congratularsi

${ }^{8}$ Le espressioni che si riferiscono alle conoscenze e alle credenze del parlante offrono notevoli potenzialità a livello pragmatico, quindi è necessario che la pragmatica partecipi al processo di interpretazione degli enunciati contenenti gli evidenziali.

9 Su questo punto, cf. Bertuccelli Papi (1990, pp. 243-247).

10 A questo proposito Searle (1969, p. 16) dice: "All linguistic comunication involves linguistic acts“. Pronunciare un enunciato vuol dire svolgere un'attività che assume un senso in una concreta situazione interazionale.

11 Come è ben noto l'atto illocutorio indica il modo in cui un enunciato viene usato, ovvero segnala la dinamica interazionale (illocutionary acts: informing, ordering, warning, undertaking, ecc.), mentre l'atto perlocutorio indica gli effetti sull'atteggiamento dell'ascoltatore ("what we bring about or achive by saying something, such as convincing, persuading, deterring, surprising or misleading"; v. Austin Lecture IX 1962, pp. 109-120). 
(congratulate). ${ }^{12}$ È chiaro che l'uso delle strategie evidenziali appartiene all'atto illocutorio di asserzione, constatazione, affermazione (" $S$ has evidence (reasons, etc.) for the truth of $p$ / It is not obvious to both $S$ and $H$ that $H$ knows $p$ ").

Nella presente analisi utilizzeremo anche la nozione di forza illocutoria (illocutionary force) che indica l'impiego di mezzi linguistici con i quali il parlante intende raggiungere gli effetti desiderati sull'ascoltatore. Per forza illocutoria si intende generalmente la forza assegnata da elementi linguistici all'interno di un dato contesto. Gli enunciati contenenti evidenziali possono assumere dei valori pragmatici riferibili a questa forza, quindi tali enunciati non esauriscono la loro importanza interazionale al livello dei loro significati (atto locutorio), né al livello degli scopi ed effetti (atto perlocutorio). Per questa forza illocutoria, secondo noi, inerente all'espressione delle strategie evidenziali, un enunciato assume uno status pragmatico. ${ }^{13}$ In questo lavoro la nozione di forza illocutoria è contenuta anche nella sua accezione estesa a sequenze dialogiche. L'ascrizione di una determinata forza a un enunciato è oggetto di interazione e scambio comunicativo fra interlocutori, ossia tale forza esibisce sfumature e gradi potenzialmente differenti in rapporto alla natura di una strategia evidenziale. ${ }^{14}$

Tenendo presenti tutti i valori semantici dei vari tipi di fonte abbiamo individuato le loro possibili implicazioni pragmatiche nelle due lingue esaminate. ${ }^{15}$

12 Sulla questione degli atti linguistici, v. Austin (1962); Searle (1969); Levinson (1983); Bertuccelli Papi (1993).

${ }^{13}$ Come già si è visto più volte, un enunciato è dotato di un significato (verofunzionale) e di una funzione comunicativa o di una forza contestuale. Attraverso gli evidenziali il parlante contribuisce alla costruzione del senso e della forza di ciò che comunica.

${ }^{14}$ A seconda della forza illocutoria, Austin (1962, p. 151), suddivide gli atti linguistici in verdictives. exercitives, commissives, behabitives, expositives. Quest'ultima classe contiene le asserzioni con evidenziali.

${ }^{15}$ Le implicazioni che seguono sono il risultato dell'analisi del corpus e degli studi dedicati ai valori pragmatici degli elementi linguistici: Searle (1969); Levinson (1983); Bertuccelli Papi (1992, 1993); Ciliberti (1992); Caffi (1992); Sbisà (1992). 
Dunque, i valori pragmatici più frequenti degli evidenziali indicanti fonti percettive e inferenziali dirette (firsthand sources) sono contenuti nei seguenti elementi: ${ }^{16}$

a. intensificatori; forza illocutoria maggiore/rafforzata/intensificata (f.i.m. $)^{17}$

b. un particolare/forte impegno persuasivo/dissuasivo (f.i.p./d.)

c. assunzione della responsabilità discorsiva (a.r.d.)

d. aumento del coinvolgimento del parlante alla veridicità della sua asserzione (a.c.p.)

e. aumento dell'affidabilità/credibilità (a.a./c.)

Gli evidenziali provenienti dalle fonti dirette possono assumere un'estensione nel contesto traducendosi in significati pragmatici citati. Come si vede, più valori pragmatici possono essere espressi da un tipo di fonte. Le fonti dirette sono caratterizzate da un'alta credibilità assegnata agli enunciati. Quindi, si usano spesso negli enunciati che hanno lo scopo di convincere o di persuadere / dissuadere (suscitando e conquistando la fiducia dell'ascoltatore). Inoltre, con i mezzi evidenziali diretti il parlante assume la responsabilità discorsiva in modo forte: in tal caso l'ascoltatore ha il diritto di contare sulla verità espressa dal parlante (Pensavo anch'io che fosse un luogo comune su quelli del sud, ma l'ho sentito con queste mie orecchie). ${ }^{18}$

Gli evidenziali come indicatori di forza illocutoria marcano le nostre asserzioni e intensificano o mitigano la presentazione dei nostri scopi pragmatici aumentando o diminuendo il coinvolgimento del parlante alla veridicità di ciò che dice e influenzando nello stesso tempo il comportamento dell'ascoltatore. Le fonti indirette (non firsthand sources) costituiscono la base per la variazione della forza pragmatica in senso attenuativo e riduttivo:

16 A nostro avviso anche l'inferenza eseguita dal parlante costituisce una fonte diretta.

17 Gli elementi intensificatori servono per aumentare la forza illocutoria di un enunciato. Nelle parentesi vi sono le abbreviazioni per ciascun valore pragmatico che accompagnerà i dati del corpus.

18 Anche in situazioni ufficiali, ad esempio, nei processi giudiziari le testimonianze dirette percettive suscitano più fiducia. 
a. diminuzione della credibilità (d.c.)

b. con funzione attenuante /mitigatori; attenuazione della forza illocutoria (a.f.i.)

c. strumenti / dispositivi dissociativi / manifestazione del distacco (s.d.)

d. riduzione del coinvolgimento del parlante alla veridicità della sua asserzione (r.c.)

e. riduzione o non assunzione della responsabilità discorsiva (r.r.)

Gli evidenziali basati sulle informazioni riportate si utilizzano maggiormente per evitare gli eventuali effetti negativi che possono derivare dall'uso di asserzioni troppo forti / determinate / risolute / sicure. Si usano anche con lo scopo di attenuare o di minimizzare gli enunciati di contenuto spiacevole, ostile o sfavorevole.

Ovviamente, l'ascoltatore è destinatario delle strategie evidenziali sia dirette che indirette.

Tali valori consentono di collocare in un quadro di analisi omogeneo e generale tutti gli enunciati a base evidenziale e offrono la possibilità di un'ulteriore suddivisione fondata su elementi di carattere graduale relativa all'affidabilità/credibilità delle informazioni: basso / medio / alto grado di $X .{ }^{19}$ Tuttavia, la gerarchia e la gradualità dei valori pragmatici degli evidenziali può essere espressa in termini binari di credibilità: credibilità alta (fonti dirette) credibilità bassa (fonti indirette).

Dal punto di vista sociolinguistico, gli interlocutori sono allo stesso tempo: $a$. membri di uno o più gruppi sociali o socioculturali, $b$. individui e $c$. partecipanti di una concreta situazione comunicativa. Questo vuol dire che anche l'uso degli evidenziali manifesta caratteristiche personali e caratteristiche determinate dallo scambio linguistico in un certo ambiente sociale. Gli evidenziali contribuiscono alla riuscita e alla appropriatezza della comunicazione linguistica visto che tramite tali mezzi ci si impegna alla verità e ad un comportamento governato da regole socioculturali. ${ }^{20}$ Per quanto riguarda la dimensione socioculturale del-

19 Il grado di affidabilità da parte del parlante riguardo la verità dell'enunciato e gli effetti possibili sull'ascoltatore forniscono interessanti spunti per approfondimenti e riflessioni linguistiche.

${ }^{20}$ I partecipanti ad un atto/evento comunicativo stipulano una sorta di accordo sociale che viene realizzato sotto forma di obblighi e di aspettative. Per questo aspetto, si veda Ciliberti (1992, p. 456). 
la conversazione, bisogna menzionare le massime di Grice, che hanno l'obiettivo di regolare il buon comportamento linguistico nella società. Dalle massime conseguono le ben note implicature conversazionali: "the maxims generate inferences beyond the semantic content of the sentences uttered. Such inferences are conversational implicatures" (Levinson, 1983, p. 103).

Nella prossima sezione procediamo all'analisi dei dati in riferimento ai suddetti valori pragmatici. Per ogni singola strategia evidenziale precisiamo i valori pragmatici ricorrenti tra le lingue in esame evidenziando i tratti comuni e quelli particolari. ${ }^{21}$

\section{ANALISI DEL CORPUS}

Nell'analisi dei dati, oltre agli strumenti categoriali e definitori della linguistica teorica e della logica, adotteremo una metodologia esplicativo-interpretativa basata sulle procedure euristiche e sull'analisi di attestazioni reali d'uso di evidenziali in repertori testuali di vari tipi. ${ }^{22}$ I risultati saranno verificati da parlanti nativi. Inoltre, utilizzeremo l'analisi contrastiva perché mettendo a confronto alcuni meccanismi evidenziali italiani e croati e classificandoli in base al loro minore o maggiore grado di equivalenza semantico-strutturale, potremo dare risposte ad alcune domande relative alle circostanze concrete d'uso degli evidenziali, ossia alla loro pertinenza pragmatica.

Le strategie evidenziali esaminate in questo lavoro sono costituite dai seguenti mezzi linguistici: avverbi evidenziali, forme verbali, congiunzioni (complementatori) e costruzioni a sollevamento.

21 Le strategie evidenziali e i rispettivi dati verranno organizzati in base al valore semantico a cui si accostano maggiormente.

22 Gli esempi sono tratti dal corpus CORIS/CODIS che è costituito da diversi sottocorpora (stampa, narrativa, prosa accademica, ecc). 


\subsection{Avverbi evidenziali}

Gli avverbi italiani e i loro equivalenti croati relativi in primo luogo all'evidenzialità sono: apparentemente (naoko, prividno, naizgled), evidentemente (očigledno), ovviamente (očito), chiaramente (jasno, očito), visibilmente (vidljivo), palesemente (očevidno, javno), manifestamente (očitovano, pokazano, očevidno, očigledno).

I valori semantici di questi avverbi in entrambe le lingue hanno una base percettiva diretta, ad esempio, apparentemente "per quanto si può vedere a un primo sguardo" (što se može vidjeti na prvi pogled / po izgledu); evidentemente "che può essere percepito attraverso i sensi" o "che è chiaramente visibile"; "di immediata perspicuità / trasparenza per la propria chiarezza sia cognitiva/logica sia sensibile" (što je očito / očevidno / očigledno). ${ }^{23}$

Esemplificazione: ${ }^{24}$

a. Questi medici della Venezia più segreta - che lavorano solo nelle ore notturne in un palazzotto apparentemente (na prvi pogled) abbandonato - sono veri e propri alchimisti. (ev. dir. visiva e inferenziale: f.i.p. / a.c.p. $)^{25}$

b. $[\ldots]$ con le gambe accavallate e le mani intrecciate attorno al ginocchio: apparentemente assorto (naoko), ma solo per poter ascoltare senza dare l'impressione di farlo. (ev. dir. visiva: f.i.m. / a.c.p.)

c. Il direttore era abbronzato, molto abbronzato, evidentemente (očito) aveva preso il sole nel parco delle terme. (ev. dir. visiva: f.i.m.) ${ }^{26}$

23 In croato anche la base della formazione è trasparente: oko / oči (= l'occhio / gli occhi). In latino: eviděntem < evidēri < ěx vidēri "mostrarsi all'esterno".

24 Questa parte del corpus è costituita da oltre 250 esempi tratti da CORIS/CODIS. Per ragioni di spazio, qui non riportiamo tutti i dati presi in esame.

25 Il parlante sa che il palazzotto non è abbandonato anche se sembra che lo sia e vuole convincerne l'ascoltatore.

${ }^{26}$ Consideriamo un altro esempio con questo avverbio di valore puramente evidenziale: [...] e vicino c'è un cofanetto pure d'avorio, contenente due minuscoli pettini e uno specchietto d'argento, evidentemente (očito/očigledno) gli oggetti da toilette della bambola (f.i.p. /f.i.m.). 
d. Guardi solo come sta lì seduto, evidentemente (očigledno) tutto preso dagli affari suoi. (ev. diretta visiva: a.c.p.)

e. Ovviamente (očito), davanti a lui nessuno ha protestato. (ev. dir. visiva: f.i.m.)

[...] ebbene il giapponese ovviamente ha subito dei mutamenti. (ev. dir. visiva: f.i.m.). ${ }^{27}$

f. Il sole era fastidioso, le sue scarpe chiaramente (očito) inadatte e nella villa c 'era troppa gente. (ev. dir. visiva: a.c.p.)

g. Lo provava chiaramente (jasno/očito) quel fumo che usciva dal camino. (ev. dir. visiva: f.i.m. / a.r.d.)

h. Tremava visibilmente (vidljivo) e mandava un odore misto di sporco e di mare. (ev. dir. visiva: a.c.p.)

i. E proprio da uno di quei balconi, ha raccontato una Gruber in giubbotto antiproiettile, visibilmente (vidlivo) provata in volto [...]. (ev. dir. visiva: a.c.p.)

j. Era palesemente (očigledno/vidljivo/očito) in imbarazzo. (ev. dir. visiva: f.i.m./ a.c.p.)

k. Palletti era palesemente (očigledno /očevidno) compiaciuto (ev. dir. visiva: f.i.m.)

1. I giocattoli, i loro componenti e le parti staccabili dei giocattoli manifestamente (očigledno) destinati a bambini di età inferiore a 36 mesi. (ev. dir. visiva: f.i.m.)

$\mathrm{m}$. Se mi veniva in mente qualche idea musicale, essa era o banale e arcinota o manifestamente (očevidno) in contrasto con tutte le leggi dell'arte. (ev. dir. inferenziale: f.i.p.). ${ }^{28}$

Analizzando i dati risulta difficile determinare nettamente i valori pragmatici precisi o univoci legati agli avverbi evidenziali poiché i loro significati sovente si sovrappongono, ovvero nello stesso momento sono impliciti più valori pragmatici rappresentativi di questo tipo di strate-

27 Il significato evidenziale e il rispettivo valore pragmatico spesso conseguono dal contesto, come nell'esempio: I progetti di espansione non sono ovviamente archiviati ("abbiamo diverse opportunità sul tavolo, le stiamo studiando", ha continuato Rondelli), (f.i.m.).

28 Nel seguente esempio: Sono decenni che l'Unione delle Camere Penali denunzia senza tregua come questa dei magistrati fuori ruolo sia una pratica malsana, perché manifestamente incompatibile con il principio fondamentale di ogni democrazia politica, ciò̀ quello della separazione dei poteri, l'avverbio manifestamente è equivalente degli avverbi croati očitovano e pokazano. 
gie evidenziali. ${ }^{29}$ La determinazione precisa del valore principale di un enunciato con questi avverbi è deducibile dal contesto. ${ }^{30}$ Comunque, possiamo dire che, nonostante le potenziali sovrapposizioni ed estensioni, le associazioni tra i valori semantici strettamente evidenziali e quelli pragmatici sono quasi automatiche, anche se certamente la definizione esatta del grado di affidabilità e di coinvolgimento nella veridicità dell'enunciato può dipendere dal contesto. L'evidenzialità visiva diretta implica un alto grado di credibilità, di convinzione da parte del parlante, di forza persuasiva/dissuasiva e di responsabilità conversazionale mentre l'evidenzialità diretta inferenziale molto spesso assume delle connotazioni riguardanti l'aumento del coinvolgimento: ad es. Soffermandomi su questa considerazione, vedo manifestamente (jasno) che non esistono indicazioni precise, nè segni abbastanza certi per mezzo dei quali sia possibile distinguere nettamente la veglia dal sonno (ev. inferenziale: alto grado di coinvolgimento = a.g. di c.).

In aggiunta, dall'esame dei dati consegue che l'avverbio visibilmente nella quasi totalità dei casi viene usato con prevalente valore evidenziale. La coerenza e la trasparenza semantica di questo avverbio hanno il compito di fornire un'interpretazione precisa del suo scopo pragmatico principale, di rafforzare ulteriormente una testimonianza già forte: Era tanto scheletrica e malmessa [...], che era visibilmente andata. L'uso dell'avverbio è caratterizzato dalla proliferazione di serie sintagmatiche molto frequenti: visibilmente malato / confuso / rinnovato / irritato / emozionato / amareggiato / rilassato / sconvolto / eccitato / in imbarazzo / con la mente altrove / affranto / commosso / assonnato / scosso / emozionato / alticcio / dimagrito. Con la stessa frequenza e gli stessi valori pragmatici appare in croato il suo equivalente vidljiv: vidljivo ljutit / iscrpljen / potresen / odmoren ecc. ${ }^{31}$

29 Inoltre, i significati evidenziali degli avverbi esaminati non di rado coincidono con i loro significati epistemici, cf. Peša Matracki \& Batinić Angster (2016, pp. 33-34).

30 Perciò, nella maggioranza dei casi, abbiamo analizzato il microdiscorso formato da due o più frasi governate da uno scopo pragmatico.

31 Una simile proliferazione si nota anche a proposito dell'avverbio manifestamente ma con prevalente valore inferenziale: manifestamente / erroneo / inammissibile / impossibile / contrario / imprudente / infondato, ecc. 
Gli avverbi chiaramente e palesemente si usano non di rado con i verbi percettivi indicanti la fonte dell'informazione (sentire, vede$r e$ ), con i verba dicendi e con quelli epistemici o espositivi, assegnando una particolare forza comunicativa all'intero enunciato: ${ }^{32}$ Non è un grido forte, ma io l'ho sentito chiaramente; [...] alcuni ghiacciai mostrano chiaramente di avere avanzato le loro fronti in epoca recente; [...] l'esistenza ha in ogni caso un garante nel metodo divino che viene rivelato palesemente nella natura, ecc. La stessa presenza del verbo percettivo non produrrebbe di per sé lo stesso effetto linguistico. Con tali verbi occorre spesso un altro avverbio con significato evidenziale più generale e con scopo rafforzativo: apertamente. È scambiabile con altri avverbi evidenziali: Galia mostrava apertamente (manifestamente / palesemente / chiaramente) la sua incredulità, pensando che sicuramente quell'nomo avrebbe potuto renderla felice.

Oltre agli avverbi, anche gli aggettivi evidenziali corrispondenti possono assumere i valori pragmatici descritti, ad es., un rancore manifesto (= visibile a tutti; očito); quest'accusa manifesta (= palese; očita); una traccia palese (= visibile sia cognitivamente sia sensorialmente; očigledan); un segno chiaro (= visibile, vidljiv). Come vediamo, il loro uso implica innanzitutto l'assegnazione di una forza persuasiva maggiore.

Se estendiamo l'analisi degli avverbi a un contesto più ampio (alla sequenza di due o più frasi prodotte da più persone), possiamo notare che il loro uso può attribuire all'atto linguistico un valore di smentita o di conferma / convincimento / giustificazione del proprio atteggiamento: E non è vero che siano mancate le donne: dai dati presentati in un convegno è emerso chiaramente che sono proprio loro ad avere maggiori opportunità di sviluppare in rete nuove professioni.

Per concludere, l'analisi ha mostrato che gli avverbi esaminati hanno pertinenza pragmatica ${ }^{33}$ visto che, tra l'altro, contribuiscono alla credibilità interazionale della conversazione / dialogo; utilizzando gli

32 In breve, fungono da intensificatori.

33 Come si è visto, gli avverbi sono più pertinenti alla dimensione pragmatica dal momento che, volendo, è possibile non usarli. 
avverbi primariamente evidenziali il parlante produce una forza pragmatica intensa e un alto grado di convincimento illocutorio acquisiti attraverso i sensi e/o l'inferenza, i quali dovrebbero persuadere l'ascoltatore (suscitando la sua fiducia) a credere alla veridicità del parlante. ${ }^{34}$ Perciò, tutti gli avverbi evidenziali a base percettiva / inferenziale ${ }^{35}$ diretta sono di solito usati quando il parlante è assolutamente sicuro dei fatti che riferisce; dunque, tali elementi linguistici sembrano esprimere un grado molto alto di affidabilità, di responsabilità e di coinvolgimento nei confronti del contenuto proposizionale.

\subsection{Forme verbali}

Anche i modi e/o i tempi verbali possono esprimere gli atteggiamenti del parlante nei confronti del contenuto proposizionale. Dal punto di vista evidenziale, l'indicativo come modo verbale di solito non è marcato pragmaticamente poiché indica la convinzione del parlante riguardante la verità del contenuto proposizionale. Il futuro, però, consente un maggiore numero di interpretazioni, una epistemica, una evidenziale ed una pragmatica.

Il condizionale con valore evidenziale in italiano e, in misura piuttosto limitata, in croato - indicando che il parlante non è la fonte originale dell'informazione contenuta nell'enunciato - può assumere diversi valori pragmatici. Tale modo verbale esprime in primo luogo la relatività e l'incertezza nei riguardi del contenuto proposizionale basato sulla non testimonialità diretta del locutore; è un segnale relativo alla credibilità/ affidabilità delle notizie riportate (cf. Peša Matracki \& Batinić Angster, 2016, p. 34).

34 Anche se il convincimento del parlante può essere non vero ma preteso, cioè in questi casi c'è sempre l'intenzionalità cosciente da parte del parlante di convincere l'interlocutore a dargli ascolto, a credere alla verità espressa nell'enunciato e di utilizzarli come strumenti di persuasione in funzione di intensificatori.

35 L'inferenza è in modo particolare presupposta negli enunciati con ovviamente, apparentemente e evidentemente. 


\section{Condizionale con esplicitazione della fonte}

Esemplificazione:

a. Secondo le voci di mercato, l'operazione verrebbe finanziata dalla casa madre Swiss Bank Corp. (d.c. / a.f.i. / r.r.)

(Prema glasinama s tržišta, operaciju je navodno platila matična kuća Swiss Bank Corp)

b. Secondo le aspettative della Funzione pubblica, la maggior parte delle domande dovrebbe giungere dal comparto degli enti locali (d.c. / a.f.i.) (Prema očekivanjima Javnih službi, najveći broj molbi trebao bi doći iz lokalnih ustanova)

c. Ma secondo i sindacati solo per 10 mila si tratterebbe di mobilità territoriale. (d.c./a.f.i.)

(Ali prema sindikatima, samo na njih 10 tisuća bi se odnosila teritorijalna mobilnost)

d. Secondo le voci londinesi il problema non sarebbero i soldi, ma la durata. (d.c. / a.f.i.)

(Prema glasinama iz Londona, problem navodno nije u novcima već u trajanju)

e. Secondo le stime ufficiali, le persone sordocieche oscillerebbero in Italia da tremila a diecimila. (d.c. / s.d. / r.r.)

(Prema službenim procjenama, u Italiji bi trebalo biti tri do 10 tisuća gluhoslijepih osoba)

Nei casi in cui la testimonialità non diretta del locutore è evidenziata tramite la menzione esplicita della fonte indiretta, i valori pragmatici più frequenti che sottostano alla semantica della scelta modale sono i seguenti: la diminuzione della credibilità; la presa di distanza dal contenuto proposizionale (strumento dissociativo); la riduzione della responsabilità nei confronti del destinatario dell'enunciazione. In effetti, gli esempi analizzati mostrano che le giustificazioni delle informazioni riportate non sono sufficienti, ovvero il possesso di dati di tipo evidenziale indirettamente accessibili non rappresenta un mezzo sufficientemente affidabile per rendere certa/vera l'informazione contenuta nell'enunciato.

L'uso del modo verbale dipende dall'atteggiamento soggettivo del locutore: se si affida alla fonte, usa solitamente l'indicativo: Secondo 
le più accreditate teorie evoluzionistiche, tutti gli esseri viventi hanno origini comuni; Secondo le scoperte degli antropologi il concetto di anima si ritrova presso tutti i popoli della terra. Qui, il senso contestuale o senso comunicativo implica un alto grado di affidabilità nella verità proposizionale. Perciò il condizionale appare più sovente negli enunciati esprimenti una fonte incerta / indefinita (secondo le voci / secondo le ipotesi / secondo i giornali / si dice): Da qui sarebbe partito per il Marocco dove, si dice, il padre avrebbe trovato il primo rifugio; Secondo i tabloid la moglie di William sarebbe in attesa di un quarto figlio. ${ }^{36}$ Tuttavia, prima di procedere in questa direzione, bisogna dire che le associazioni tra il tipo di fonte e l'uso del condizionale non sono automatiche ma, a volte, determinate dalla dinamica interazionale, ad es., Secondo la prognosi dei medici, dovrebbef?'deve cavarsela in una decina di giorni (Prema predviđanjima liječnika, trebao bi/treba ozdraviti za desetak dana).$^{37}$

I valori pragmatici aggiuntivi del condizionale nei suddetti casi si riflettono nella bassa frequenza d'uso del condizionale negli enunciati in cui la fonte viene esplicitata e considerata affidabile (credo che secondo $X, p$ è vero). Infatti, della totalità dei casi del corpus esaminato, circa un quarto contiene il condizionale, mentre i rimanenti presentano il verbo al modo indicativo: Sempre secondo le ricerche americane, $i$ bimbi nati da madri che soffrivano di gengivite sono più esposti ad alcuni disturbi di salute tipici dei piccoli nati da mamme fumatrici.

Gli enunciati contenenti questa fonte indiretta (secondo le ricerche didattiche / della studiosa / degli esperti / scientifiche, ecc.) sono prevalentemente all'indicativo (dei 15 casi esaminati: 3 condizionali e 12 indicativi; cioè nell' $80 \%$ dei casi è usato l'indicativo).

36 L'esempio tratto dal sito: www.donnaglamour.it. Come è ben noto, il condizionale segnala le notizie sentite/lette ma non verificate; a volte viene usato anche quando si ha la conferma dell'informazione: In particolare il Daily Mail ha confermato che lo staff della Duchessa di Cambridge che cura la sua immagine sarebbe decisamente infastidito dalle scelte di look di Meghan Markle (www.cosmopolitan.com).

37 L'esempio originale italiano è con il condizionale dovrebbe cavarsela. Secondo il giudizio dei parlanti nativi intervistati l'indicativo deve non è ammesso. Invece, tale forma (treba) in croato è accettabile. 
Ora consideriamo alcuni esempi senza indicazione esplicita della fonte dell'informazione:

Lennie sarebbe nato e qualcuno avrebbe avuto cura di lui. (d.c. / a.f.i. / r.r.) (Lennie je navodno rođen i netko se brine za njega)

Il ragazzo prodigio di Londra avrebbe costruito la sua ricchezza a suon di scommesse vincenti al Liffe. (d.c. / s.d. / a.f.i.)

(Londonsko čudo od djeteta navodno je stvorilo svoje bogatstvo na opkladama)

Il missile sarebbe partito da un lanciatore Buk. (d.c. / a.f.i. / r.r.)

(Raketa je navodno ispaljena s lansirališta Buk)

L'astrofisico lavorerebbe per una potenza straniera (d.c. / a.f.i. / r.r.)

(Astrofizičar navodno radi za jednu stranu silu)

Come si può vedere dagli esempi italiani e dalla comparazione con il croato, il condizionale italiano ha valore più strettamente evidenziale nelle frasi in cui la fonte non è espressa esplicitamente (informazione non prodotta dal parlante). Tenendo conto di tutte le varie interpretazioni pragmatiche dei dati, possiamo identificare i seguenti valori ed effetti pragmatici: i. grado di affidabilità tra basso e medio; ii. coinvolgimento è piuttosto ridotto; iii. non-assunzione della responsabilità discorsiva, cioè una presa di distanza dalla verità della notizia riportata. Quando si tratta dell'ambito giornalistico il condizionale esprime il rispetto degli obblighi e delle aspettative, il quale costituisce una delle caratteristiche essenziali di un buon servizio pubblico, un buon addetto stampa, un buon giornalista, un buon giornale, ecc. La scelta del modo verbale esprime quindi un atteggiamento del parlante nei confronti delle notizie / conoscenze / credenze riportate, vale a dire il valore comunicativo degli enunciati contenenti il condizionale con valore evidenziale è costituito dal commento sul valore della veridicità del contenuto comunicato e dai rispettivi effetti pragmatici.

In croato, l'uso del condizionale con valore evidenziale in analogia con quello italiano è molto raro e stilisticamente marcato ('Raketa bi bila ispaljena s lansirališta Buk; cf. Gnjatović \& Matasović, 2010, p. 91). Come vediamo dalle traduzioni in croato, il condizionale si esprime con l'avverbio navodno (ingl. reportedly / allegedly). In italiano non 
esiste un avverbio equivalente, ma il suo valore può essere indicato da alcune espressioni come secondo quanto (stando a quanto) riferito, secondo quel che si dice, a quanto si dice, a detta di: un Evangeliario greco che, a quanto si dice, sarebbe appartenuto a san Giovanni Crisostomo (= koji je navodno pripadao...); ${ }^{38}$ Secondo quanto riferito ieri notte il gruppo sarebbe pronto (= je navodno spremna) a rilevare i titoli Agf versando 320 franchi per azione. Partendo dall'inglese e dal croato questi avverbi sono generalmente tradotti in italiano con presumibilmente (= prevedibilmente, probabilmente): notizia presumibilmente vera (= navodno istinita vijest). ${ }^{39}$

L'avverbio croato navodno in primo luogo indica la fonte non diretta (di tipo hearsay) che naturalmente implica sia l'incertezza o la probabilità (modalità epistemica) sia i sopraddetti valori pragmatici mentre presubilmente esprime primariamente la probabilità ossia la modalità epistemica: "secondo quanto si può presumere" (= che può essere ritenuto vero) che equivale al "che può essere ragionevolmente congetturato o supposto": Mia zia è presumibilmente in Nuova Zelanda. Ma, nella frase C'è presumibilmente un orso nella zona, il valore dell'avverbio è maggiormente evidenziale e questo implica un grado più alto di affidabilità. ${ }^{40}$

${ }^{38}$ Per quanto riguarda le forme verbali che appaiono in costruzioni con queste espressioni, vi sono sia dei predicati all'indicativo che al condizionale: A quanto si dice, comunicano con lui lasciandogli messaggi sotto una lapide in un minuscolo cimitero di Pitcairn Island; E ai saccheggi, secondo quanto riferito, parteciperebbero anche agenti di polizia municipale.

39 Per il croato abbiamo consultato il dizionario di Deanović \& Jernej (1997); per l'inglese vari dizionari online, ad es., Collins Online Dictionary, Oxford English Dictionary, wordReference.com.

${ }^{40}$ Vuol dire "qualcuno l'ha visto". In sostanza, tale avverbio può avere un'interpretazione inferenziale "dato ciò che so / in base alle mie conoscenze, presumo che p". 


\section{Futuro}

Il valore evidenziale del futuro italiano e croato è solitamente di tipo inferenziale-congetturale. Pragmaticamente è interpretabile soprattutto come uno strumento dissociativo dal contenuto dell'informazione espressa:

(4)

No - risponde - ma sui giornali se ne è parlato, la stampa avrà avuto qualche soffiata. (r.c.)

(Ne - odgovara - ali u novinama se o tome govorilo, bit će da im je netko dojavio)

Be ', pensai, a quest'ora probabilmente sarà morta. (r.c.)

(Pomislih, bit će da je sada već mrtva)

Però il ministro avrà visto il suo film. Non lo so. ( r.c.)

(Ali, bit će da je ministar vidio njegov film)

Non si sente nulla. Tutti saranno andati via. (r. c.)

(Ne čuje se ništa. Bit će da su svi otišli)

Il valore pragmatico aggiuntivo del futuro è sottinteso maggiormente nell'atto linguistico della domanda, esplicita e implicita: Domanda: sarà riuscito il premier a vedere la puntata natalizia di AnnoZero?

Dai dati consegue che il futuro con valore evidenziale di tipo inferenziale-congetturale riguarda elementi linguistici più ampi della frase e nella maggioranza dei casi solo in tali minidiscorsi è interpretabile pragmaticamente: Non tollera e finge di non saper riconoscere le «cavolate» di Enzo Jannacci. Sarà stato soltanto un caso? Il futuro lascia aperta l'alternativa esclusiva: vero vs. falso, perciò un enunciato contenente il futuro inferenziale è spesso accompagnato dalla frase non so / non lo so. Si tratta altresì di un atteggiamento personale pronto a credere che il contenuto proposizionale sia vero senza impegnarsi nei confronti della sua veridicità: Lo scorso fine settimana ho dormito bene come non mi succedeva da un po'. "Sarà stato il whisky». Cerco di buttarla sul ridere perché non so cos'altro fare. Questo minidiscorso non avrebbe le stesse sfumature e connotazioni pragmatiche se avessimo usato un altro tempo.

Con le strategie evidenziali basate sull'attività sensoriale i valori pragmatici del futuro sono associabili all'intensificazione della forza il- 
locutoria che proviene proprio dall'alternativa tipica del futuro (vero falso): Lorenzo, che avrà avuto quindici anni, le fece un gesto con la mano, duro, senza replica (poteva avere anche meno di quindici anni). ${ }^{41}$

Nei contesti di tipo evidenziale può apparire il modo congiuntivo, soprattutto con l'espressione perifrastica 'si dice che' con valore di reportedly e allegedly: Di tutti si dice che il denaro venga loro dalla benevolenza del potere politico (O svima se govori da im bogatstvo navodno dolazi od naklonosti političke moći). Nelle costruzioni analoghe senza congiuntivo, nella traduzione in croato non c'è navodno: In genere si dice che un carattere è rappresentato da un grafema ${ }^{42}$ (Općenito se kaže da grafem predstavlja slovo). Le costruzioni con congiuntivo sono marcate pragmaticamente dato che esprimono una riduzione del coinvolgimento nella veridicità dell'enunciato.

Un'altra costruzione con il verbo modale dovere (ingl. must; cro. morati) può assumere un parziale valore evidenziale di tipo inferenziale: Si è trasferito in un altro continente per starle vicino. Deve amarla moltissimo (Preselio se na drugi kontinent da bude s njom. Mora da je jako puno voli). Qui la convinzione e la forza persuasiva è di grado superiore rispetto ai casi citati precedentemente. In croato, la negazione della frase con il valore evidenziale del verbo reggente dovere si realizza mediante l'aggiunta della particella negativa ne (non) al verbo retto (amare): Mora da je ne voli jako puno. In italiano invece tale particella può precedere sia il verbo reggente sia quello retto: $i$. Non deve amarla moltissimo, ii. Deve non amarla moltissimo. Secondo i parlanti nativi intervistati la posizione della negazione cambia la sfumatura di significato di queste frasi. La prima $(i$.), oltre al valore evidenziale, può avere anche quello deontico (É necessario che non la ami moltissimo; Ne smije je jako puno voljeti), mentre nella seconda (ii.) dovere ha solamente il valore evidenziale di tipo inferenziale (E molto probabile che non la ami moltissimo).

${ }^{41}$ Il che rendeva il suo gesto ancora più sorprendente. L'esempio è tratto da CORIS/ CODIS, dal sottocorpus MON 2005_07.

${ }^{42}$ Con si dice è più frequente l'indicativo: Per lo più si dice che fu uccisa da Eracle. 
L'interpretazione del deve essere è che il parlante non è assolutamente convinto/certo delle sue parole perché non ha l'evidenza diretta per la sua affermazione: Perché crede che spalanchi tanto gli occhi? Deve aver visto qualcosa, da bambino. Nei contesti comunicativo-situazionali analoghi, l'implicatura conversazionale consiste nella seguente scelta del parlante: le prove, gli evidenziali contano di più della valutazione epistemica (nonostante l'alto grado di coinvolgimento nella veridicità di ciò che dice il parlante). ${ }^{43}$

In generale, le strategie evidenziali delle suddette forme verbali si usano in relazione alle convinzioni (ridotte /non forti) o ai gradi di dubbio sulla verità del contenuto proposizionale e questi elementi costituiscono il nucleo semantico intorno al quale si sviluppano le potenzialità interpretative sul piano pragmatico.

\subsection{Uso del complementatore}

In entrambe le lingue ci sono costruzioni sintattiche subordinative che indicano alcuni tipi di evidenzialità. Si tratta in primo luogo delle costruzioni con i verbi di percezione, che suddividiamo in due gruppi: a. quelle introdotte dalla congiunzione che $(\mathrm{kako} / \mathrm{da})$, denominata complementatore ${ }^{44} b$. frasi senza introduttori. In croato queste ultime sono rarissime.

\section{Frasi con complementatore:}

Lo vedo che scappa in stanza! (f.i.m. /a.c.p. /a.a.c.)

(Vidim ga kako/da bježi u sobu)

Sono già due volte questo mese che ti vedo bere troppo. (f.i.m. /a.c.d. /a.a.c.)

(Već dva puta ovaj mjesec vidim da previše piješ)

Sento che le lacrime ricominciano a bagnarmi le guance. (f.i.m.)

(Čujem kako/da mi suze ponovno klize niz obraze)

43 Sulle analisi delle costruzioni col verbo dovere (ing. must, ted. moeten), si veda De Haan (1999).

44 Questo nome è stato introdotto per indicare il tipo di rapporto tra introduttore di frase e la frase stessa: la frase flessa funge da complemento della testa funzionale che (cf. Graffi, 1994, p. 195). 
La percezione diretta in croato può essere espressa tramite due operatori, kako e $d a$, che implicano sfumature semantiche diverse attorno alle quali si possono condensare diversi valori pragmatici: le costruzioni con $d a$ esprimono una forza illocutoria di grado più intenso..$^{45}$

I dati mostrano che sia in italiano che in croato queste costruzioni esprimono una forza illocutoria di grado molto elevato e assegnano agli enunciati valore di atti linguistici indiretti: di smentita / conferma / consiglio / ordine, ad es., lo vedi che scappa può implicare "Prendilo!"; sono gia due volte che ti vedo bere "Devi smettere di farlo!".

Queste costruzioni sono utilizzate molto efficacemente per comunicare con altri, per organizzare dialoghi e per strutturare diversi scopi pragmatici: É la terza battuta che ti sento fare in sei anni / Dovresti farle più spesso; È la prima volta che ti sento fare questo elogio della verità / La verità merita di più. L'efficacia comunicativa e persuasiva/ dissuasiva di questi enunciati non sarebbe la stessa senza verbi percettivi (è la terza battuta che dici/fai / è la prima volta che fai...). ${ }^{46}$

Anche il verbo guardare, "rivolgere lo sguardo su qualcuno/qualcosa", denota una percezione diretta:

(6)

Mi guarda mentre passo tra i tavoli con il grembiulino nero. (f.i.m.)

(Gleda me kako prolazim među stolovima s crnom pregačicom)

Guarda che ti vedo che scrivi invece di lavorare. (f.i.m.)

(Pazi, vidim te da pišeš umjesto da radiš)

Guarda come mi sono ridotto a vivere. (f.i.m.)

(Gledaj kako sam nisko pao u životu)

In entrambe le lingue la percezione diretta può essere espressa tramite vari operatori che implicano connotazioni semantiche e pragmatiche diverse: come / kako e mentre / dok hanno un valore più descrittivo

45 Secondo l'analisi di Gnjatović \& Matasović (2010) e anche secondo le valutazioni dei parlanti nativi.

${ }^{46}$ I parlanti nativi sia italiani che croati hanno confermato la suddetta affermazione. 
di che e una forza illocutoria non particolarmente intensa, ${ }^{47}$ anche se spesso esprimono un'immediatezza evidenziale di prim'ordine. ${ }^{48}$

\section{Senza complementatore: ${ }^{49}$}

Con grande sollievo vedo arrivare la macchina di Landon. (f.i.m.)

Paola, sei così bella! E vedo il tuo viso cambiare. (f.i.m.)

Mi sento suonare nelle orecchie le parole dei miei sogni. (f.i.m.)

Dagli esempi risulta che le costruzioni percettive in italiano con e senza complementatore esprimono lo stesso tipo di evidenzialità: quella diretta basata sui sensi, ma si distinguono pragmaticamente: secondo gli informatori le costruzioni in (7) possiedono una forza pragmatica di massimo grado.

\subsection{Sollevamento}

Adesso prendiamo in analisi le costruzioni a sollevamento (ingl. raising; cro. podizanje) del soggetto:

Mi sembra che il cancello sia aperto. / Il cancello mi sembra aperto.

(Čini mi se da su vrata otvorena; Vrata mi se čine otvorena/otvorenima)

Sembra che il sentiero sia rischiarito. / Il sentiero sembra rischiarito.

(Čini se da je staza svjetlija; Staza se čini svjetlijom)

Sembra che il pianoforte nell'angolo sia abbandonato. / Il pianoforte nell'angolo sembra abbandonato.

(Čini se da je klavir u uglu napušten; Klavir u uglu se čini napuštenim)

47 Secondo le valutazioni dei parlanti nativi, un po' meno intensa di quella ottenuta con che.

48 In croato la scelta del complementatore può indicare un'evidenzialità di tipo diverso. In dipendenza del complementatore che, con i verbi čuti (sentire) e vidjeti (vedere) è possibile la duplice interpretazione (diretta e indiretta) dell'evidenzialità espressa. Invece, con i complementatori kako e dok, l'interpretazione è univoca, si veda Peša \& Batinić Angster (2016, p. 40).

49 Le costruzioni di questo tipo, in croato, non sono grammaticali. 
Anche la costruzione a sollevamento esprime tratti di un'evidenzialità più diretta di quella con il complementatore. In entrambe le lingue costruzioni di significato simile possono ugualmente esprimere un grado diverso di immediatezza della percezione (cognitiva e sonsoriale): L'albergo sembra (essere) aperto (= a quanto vedo); Sembra che l'albergo sia aperto (a quanto vedo o a quanto si dice). Quindi, nel primo esempio il grado di affidabilità è più alto e questo si vede dai contesti più ampi dove il tipo e il grado di esperienza percettiva sono determinati con precisione: Potremmo uscire di qua, non è molto alto ed il cancello sembra aperto (la probabilità che sia aperto è molto alta, solo un grado in meno rispetto al Vedo il cancello aperto).

\section{CONCLUSIONE}

Si è mostrato che lo status pragmatico delle strategie evidenziali risiede nell'interpretazione che si estende alla loro funzione comunicativa all'interno di un dato contesto. Dall'analisi del corpus CORIS/CODIS consegue che il sistema evidenziale delle lingue esaminate, in determinate occorrenze contestuali e a partire da determinati scambi linguistici, assolve funzioni pragmatiche ben individuabili ed esibisce una chiara preminenza empirico-cognitiva, il che conferma l'ipotesi di partenza.

Dunque, usando gli evidenziali non solo si esprimono fonti di informazione ma si comunicano fatti e notizie che producono un determinato effetto conoscitivo sul destinatario, una forza persuasiva, un impegno alla verità soggetti a giudizio/valutazione di riuscita e di appropriatezza di tipo personale e socioculturale. Le strategie evidenziali producono una certa influenza anche sull'economia relativa alla comunicazione: poiché le associazioni tra il tipo di fonte e i possibili effetti pragmatici sono quasi automatiche, possiamo strutturare le nostre conversazioni in modo più efficace e sistematico e acquisire nuovi elementi conoscitivi in modo più vantaggioso. Inoltre, come parte costituente degli obblighi di chi informa e delle aspettative di chi ascolta, il sistema evidenziale stabilisce le proprietà concettuali che concorrono a definire che cosa è un buon servizio pubblico, un affidabile parlante / oratore / giornalista / politico, ecc. 
Infine, gli evidenziali concorrono in modo importante a rafforzare e restituire il carattere eventivo o di attività della comunicazione linguistica.

\section{BIBLIOGRAFIA}

Aikhenvald, A.Y. (2004). Evidentiality. Oxford: Oxford University Press. Alai, M. (2013). Conoscenza, verità, giustificazione epistemica. In A. Livi (Ed.), La certezza della verità (pp. 31-47). Roma: Leonardo da Vinci. Austin, J.L. (1962). How to do things with Words. In J.O. Urmson \& M. Sbisà (Eds.), Cambridge/Massachusetts: Harvard University Press.

Bertuccelli Papi, M. (1990). Il significato della semantica e il senso della pragmatica. In G. Gobber (Ed.), La linguistica pragmatica (pp. 243-264). Roma: Bulzoni.

Bertuccelli Papi, M. (1993). Che cos'è la pragmatica. Milano: Bompiani.

Caffi, C. (1990). Il concetto di coinvolgimento nella linguistica pragmatica. In G. Gobber (Ed.), La linguistica pragmatica (pp. 267-299). Roma: Bulzoni.

Ciliberti, A. (1990). Uso di 'evidenziali' nelle sequenze richiesta-risposta in incontri di servizio. In G. Gobber (Ed.), La linguistica pragmatica (pp. 455-474). Roma: Bulzoni.

Collin, F., \& Guldmann, F. (2005). Meaning, Use and Truth. Burlington: Ashgate.

De Haan, F. (1999). Evidentiality and Epistemic Modality: Setting boundaries. Southwest Journal of Linguistics, 18(1), 83-101.

De Haan, F. (2001). The Relation between Modality and Evidentiality. In M. Reis \& R. Müler (Eds.), Modalität und Modalverben im Deutschen (pp. 201-216). Hamburg: Helmut Buske.

Diewald, G., \& Smirnova, E. (Eds.). (2010). Linguistic realization of evidentiality in European languages. Berlin/New York: De Guyter.

Dummett, M. (1981). Frege. London: Duckworth.

Gnjatović, T., \& Matasović, R. (2010). Evidencijalne strategije u hrvatskom jeziku. In M. Birtić \& D. Brozović Rončević (Eds.), Zbornik radova znanstvenoga skupa s međunarodnim sudjelovanjem, Drugi hrvatski sintaktički dani (pp. 89-99). Zagreb: IHJJ.

Gobber, G. (Ed.). (1990). La linguistica pragmatica. Roma: Bulzoni. 
Goldman, A.I. (1986). Epistemology and Cognition. Cambridge/Massachusetts: Harvard University Press.

Graffi, G. (1994). Sintassi. Bologna: il Mulino.

Levinson, S.C. (1983). Pragmatics. Cambridge/New York: Cambridge University Press.

Livi, A. (Ed.). (2013). La certezza della verità. Roma: Leonardo da Vinci.

McCready, E., \& Ogate, N. (2007). Evidentiality, Modality and Probability. Linguistics and Philosophy, 30(2), 147-206.

Palmer, F.R. (1986). Mood and Modality. Cambridge: University Press.

Peša Matracki, I., \& Batinić Angster, M. (2016). Evidenzialità in italiano e in croato. Studia Romanica et Anglica Zagrabiensia, LXI, 27-45.

Sbisà, M. (1990). Atti linguistici ed espressione di affetto. In G. Gobber (Ed.), La linguistica pragmatica (pp. 353-379). Roma: Bulzoni.

Searle, J.R. (1969). Speech acts. Cambridge/New York: Cambridge University Press.

Squartini, M. (2005). L'evidenzialità in rumeno e nelle altre lingue romanze. Zeitschrift für romanische Philologie (ZrP), 121(2), 246-268.

Steup, M., \& Sosa, E. (2005). Contemporary debates in Epistemology: Malden/Oxford: Blackwell.

\section{Fonti}

Corpus CORIS/CODIS (annotated version 2017) $=$ Corpus di Riferimento dell'Italiano Scritto / Corpus Dinamico dell'Italiano Scritto. Retrieved from corpora.ficlit.unibo.it.

Deanović, M., \& Jernej, J. (1997). Vocabolario italiano-croato. Zagreb: Školska knjiga.

Dizionario enciclopedico, versione online: treccani.it.

Sabatini, F., \& Coletti, V. (2008). Dizionario italiano Sabatini Coletti. Firenze: Giunti.

Riassunto: Gli evidenziali esprimono l'origine dell'informazione di cui si è in possesso, il che concerne altresì l'atteggiamento del parlante nei confronti del contenuto proposizionale. Nel presente contributo, dopo aver definito l'oggetto delle nostre riflessioni e aver delineato i vari tipi di strategie evidenziali, analizzeremo i valori pragmatici delle espressioni linguistiche che producono diversi gradi di coinvolgimento del parlante verso le sue asserzioni che, a loro volta, rappresentano atti linguistici con cui si sottolinea la verità dell'enunciato. Gli evidenziali sono spesso usati per ridurre il grado di responsabilità riguardo all'attendibilità delle asserzioni, ossia, una strategia evidenziale 
si può presentare come un tipico dispositivo dissociativo, una risorsa discorsiva a cui si fa ricorso per evitare gli eventuali effetti negativi che possono derivare dall'uso di asserzioni troppo forti. Per definire la classe degli evidenziali intendiamo rifarci agli studi svolti sia nell'ambito della linguistica che in quello della logica di giustificazione. L'obiettivo del presente lavoro è di mostrare che tali nozioni possono essere descritte in modo adeguato solo prendendo in considerazione l'analisi dei loro effetti pragmatici all'interno di un concreto evento comunicativo e sociale.

Parole chiave: evidenziali, valori pragmatici, atti linguistici, lingua italiana, lingua croata 